\title{
Taenia solium Taeniasis and Cysticercosis Control and Elimination Through Community-Based Interventions
}

\author{
Hélène Carabin • Aminata A. Traoré
}

Published online: 27 September 2014

(C) Springer International Publishing AG 2014

\begin{abstract}
Taenia solium was declared potentially eradicable by the International Task Force for Disease Eradication in 1992. Yet, very few well-designed community-based randomized controlled trials have been conducted to measure the effectiveness of alternative control strategies. Most strategies have been tested in pre-post intervention designs in very few communities, often without a control group. The only two community-based randomized controlled trials suggest that an educational program alone or a combination of human and porcine mass treatment reduce porcine cysticercosis in the short-term. A transmission dynamics model suggests that improved sanitation and pig management are more effective and sustainable than pig vaccination, or human or porcine mass treatment. Current evidence does not support the eradication of $T$. solium in the foreseeable future. Investigators should follow international recommendations on the conduct of community-based randomized control trials to provide more valid estimates of the effect and cost effectiveness of alternative control strategies for cysticercosis.
\end{abstract}

Keywords Taenia solium · Cysticercosis ·

Neurocysticercosis $\cdot$ Intervention trials $\cdot$ Community-based trials $\cdot$ Porcine $\cdot$ Human $\cdot$ Review

\section{Introduction}

The International Task Force for Disease Eradication (ITFDE) of the World Health Organization (WHO) declared Taenia

H. Carabin $(\bowtie) \cdot$ A. A. Traoré

Department of Biostatistics and Epidemiology, University of

Oklahoma Health Sciences Center, Oklahoma City, OK 73104, USA

e-mail: helene-carabin@ouhsc.edu

A. A. Traoré

e-mail: aminata-a-traore@ouhsc.edu solium as being potentially eradicable in 1992 [1]. In 2013, the ITFDE re-emphasized their opinion that $T$. solium should be considered eradicable, with the most effective method to interrupt the life cycle being improvement of human sanitation and hygiene, combined with surveillance of porcine infection through tongue inspection [2••]. Many others have made recommendations on the best approaches to control cysticercosis [3-6], but these are not based on evidence arising from well-designed community-based randomized controlled trials,

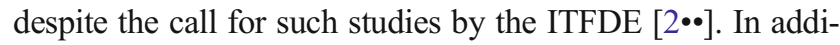
tion, no cost-effectiveness or cost-benefit analyses have been conducted to compare alternative control strategies for the simultaneous control of porcine and human cysticercosis $[2 \cdot \bullet]$. This article is a review of evidence from published community-based trials that used at least a pre-post intervention design to estimate the effect of at least one control strategy on the community-level frequency of porcine or human cysticercosis, neurocysticercosis, or human taeniasis.

\section{How to Break the Taenia solium Life Cycle}

When discussing alternative control measures for cysticercosis, it is essential to understand the life cycle of T. solium and how different types of interventions may break that cycle (Fig. 1). Briefly, humans serve as the definitive host, usually harboring one adult hermaphrodite parasite in their intestine, a condition called taeniasis. When humans with taeniasis defecate in the environment, they may shed up to five parasite segments per day, each containing tens of thousands of eggs [7, 8]. Interventions that stop the eggs from reaching the environment include the use and proper maintenance of latrines or treatment of taeniasis cases with praziquantel or niclosamide, followed by safe disposal of feces after treatment. Very little is known about factors that affect the survival of the eggs in the environment. In one experimental study, exposure to very high temperatures 


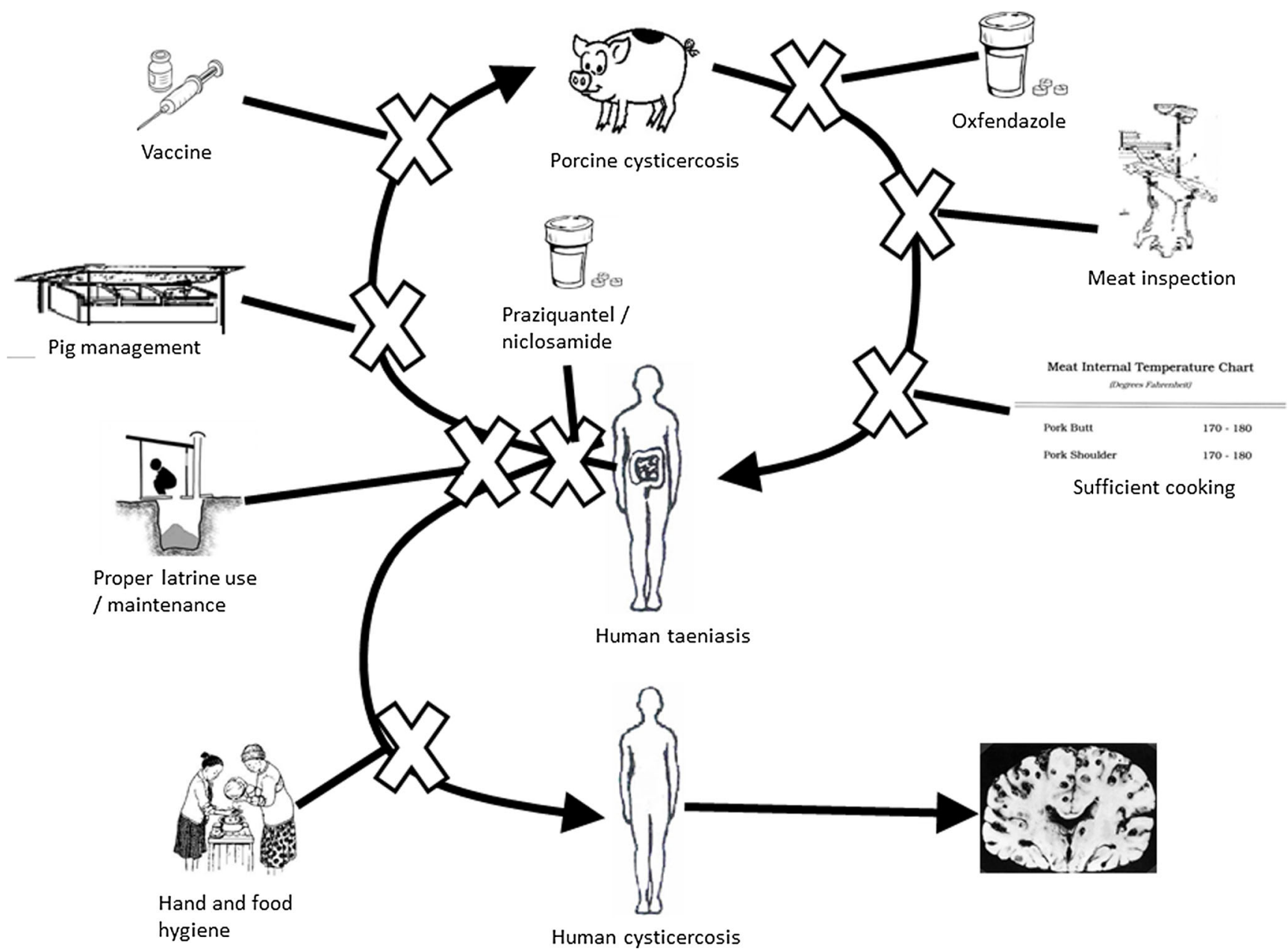

Fig. 1 Life cycle of Taenia solium cysticercosis and possible interventions for control. $X$ denotes areas where the life cycle may be interrupted

achieved $100 \%$ inactivation of T. solium eggs, and acid $\mathrm{pH}$ combined with dryer environments increased inactivation [9]. This suggests that eggs are likely to survive for months in an alkaline soil in humid environments. Pigs are the only intermediate hosts for T. solium. In areas where they are traditionally raised and left roaming, they eat human feces or food contaminated with T. solium eggs. Even penned pigs may have access to infectious eggs, when fed with contaminated food or when directly fed human feces, as reported in some endemic communities [10-12]. Interventions focusing on improved pig management practices aim at reducing access to human feces by pigs. Vaccines aim at protecting pigs from acquiring the infection when exposed to the eggs. Once ingested, the egg releases an embryo or oncosphere, which migrates primarily to the muscles where it matures into a larval stage named metacestode (commonly referred to as cysticercus). Cysticerci establish as cysts 10-12 weeks after ingestion. When humans consume undercooked infected pork meat, they develop taeniasis, and after about 2 months, worms produce eggs that are passed with feces, thus completing the cycle. Treatment of pigs, appropriate meat inspection, and sufficient cooking of pork meat are alternative strategies to stop humans from consuming contaminated meat. The public health burden of T. solium lies in the infection of humans with the larval stages, a condition called cysticercosis. Humans may develop cysticercosis when eating food contaminated with the eggs present in the environment, through autoinfection if they have taeniasis, or through direct contact with a carrier. In addition to proper use of latrines and treatment of human taeniasis carriers mentioned above, proper hand washing and food hygiene can prevent humans from acquiring cysticercosis. When the larva(e) lodge in the central nervous system, neurocysticercosis develops. Neurocysticercosis is very pleomorphic in its manifestations but seizures and epilepsy, severe chronic headaches, and focal deficits have been shown to be the most common among symptomatic cases [13].

\section{Literature Search Method and Findings}

A search of MEDLINE using the Mesh terms "taenia solium" or "cysticercosis" and publication type "randomized controlled trial" returned 48 hits. The search did not restrict for date or language. When the keyword "community" was added, only three articles remained, among which only one 
was a community-based randomized controlled trial, which was conducted in Tanzania [14]. Given these search results, we kept the mesh terms and combined them with the keywords (control OR intervention OR treatment OR vaccination OR trial) AND (community OR village* OR municipality OR household OR farm). This resulted in 163 articles, of which ten reported on original studies assessing the effect of some intervention implemented at the community level on the frequency of porcine or human cysticercosis, neurocysticercosis, or human taeniasis [14-23]. We also identified several documents on cysticercosis control, including guidelines [3, 4], book chapters [6], and review or opinion papers [5, 24]. The review of these resulted in identifying two additional articles $[25,26]$. We also found a review paper on control strategies reported in the Chinese literature [28], but it included too few details regarding methods to be included in this review. Finally, a Mexican study published after we had conducted our literature search was also included [27].

In this article we review and critique these studies according to the interventions being evaluated. Tables 1, 2, 3, and 4 describe and provide a summary of biases of studies focusing on a human outcome (Tables 1 and 3) and a pig outcome (Tables 2 and 4), respectively, to assess the effect of the intervention. None of the interventions solely targeting pigs (treatment or vaccine) assessed the effectiveness of the control strategy on human outcomes.

\section{Effectiveness of Mass Drug Administration of Antiparasitics to Humans as the Sole Intervention}

Both praziquantel and niclosamide are on the WHO essential drugs list [29]. The effect of mass drug administration (MDA) of praziquantel or niclosamide on reducing the prevalence of taeniasis and human or porcine cysticercosis has been evaluated in three community-based studies in Guatemala [17] and Mexico $[15,16]$. Yet, no randomized clinical trials estimating the efficacy of these drugs to treat taeniasis could be found in MEDLINE. It may be that the absence of a gold standard to diagnose taeniasis makes it difficult to know who is infected, leading to a challenge in assessing efficacy. An estimated efficacy of $77.9 \%$ (95\% CI 66.7-86.2) of niclosamide is probably the best available [30]. According to a review [31], the efficacy of praziquantel $(5 \mathrm{mg} / \mathrm{kg}$ ) ranges between 67 and $94 \%$, while a dose of $10 \mathrm{mg} / \mathrm{kg}$ would be $100 \%$ efficacious, but the assessment of taeniasis in these studies was problematic. The three reviewed community-based studies used a prepost intervention prevalence comparison design without a control group or randomization and were conducted in one $[15,16]$ and two villages [17]. While the authors suggested that MDA could reduce taeniasis and cysticercosis in the short- or long-term, only one study used a 42-month followup [16]. Taeniasis was measured with coproscopy and copro- antigen [32] tests in two studies [16, 17], while only coproscopy, which has low sensitivity to detect $T$. solium [33], was used in the remaining study [15]. The presence of antibodies to $T$. solium antigens, as suggestive of human cysticercosis, was measured by enzyme-linked immunoelectrotransfer blot (EITB) [34] in one study [16] and by enzyme-linked immunosorbent assay (ELISA) [35], which has poor specificity and mediocre sensitivity [36], in another [15]. Two studies [16, 17] used EITB [34] to determine antibody seroprevalence for porcine cysticercosis. All studies found a reduction in taeniasis prevalence in the shortterm, although it was not always statistically significant, with prevalence proportion ratios (PPRs) of 0.47 (95\% CI 0.20 1.11) after 6 months [16], 0.28 (95\% CI 0.15-0.53) after 10 months [17], and 0 (95\% CI undefined) after 12 months [15]. In the longer term, a PPR of 0.44 (95\% CI 0.13-1.47) after 42 months was obtained, but this study used data in which $83 \%$ of the study population pre-treatment was lost to follow-up [16]. When only those followed up for 42 months were included, a reduction in prevalence was observed, but there was insufficient information to estimate a PPR. The PPR of human cysticercosis could be estimated at 1.77 (95\% CI 1.38-2.29) 6 months post-MDA, at 0.39 (95\% CI 0.17-0.88) at 42 months using the EITB [16], and at 0.65 (95\% CI 0.39 $1.10)$ at 12 months using the antibody-detecting ELISA test [15]. The authors could not provide a reasonable explanation for the increase in human seroprevalence after 6 months, which was also observed when only including those followed up for 42 months [16]. No such increase was observed in the seroprevalence of porcine cysticercosis. Indeed, the PPR of MDA on porcine cysticercosis could be estimated at 0.46 (95\% CI 0.21-1.04) after 6 months, 0.13 (95\% CI 0.09 0.20 ) after 10 months, and 0.70 (95\% CI 0.32-1.53) after 42 months $[16,17]$. One important limitation of the study by Allan et al. [17] is that sampled pigs were considerably younger post-intervention, introducing a considerable confounding bias that could increase the magnitude of the effect.

To summarize, although these studies suggest some effect of MDA on taeniasis and cysticercosis, they all present important biases, most of which are summarized in the last section of this review. Another important limitation is the lack of assessment of the effect of repeated MDA. In conclusion, given the poorly designed nature of the studies, we have very little scientific evidence to support that community-based MDA is sufficient to generate an effective control of T. solium.

\section{Effectiveness of Mass Drug Administration of Anti-Parasitic Treatment to Pigs as the Sole Intervention}

We were unable to identify studies where anti-parasitic MDA to pigs was the sole intervention implemented at the 


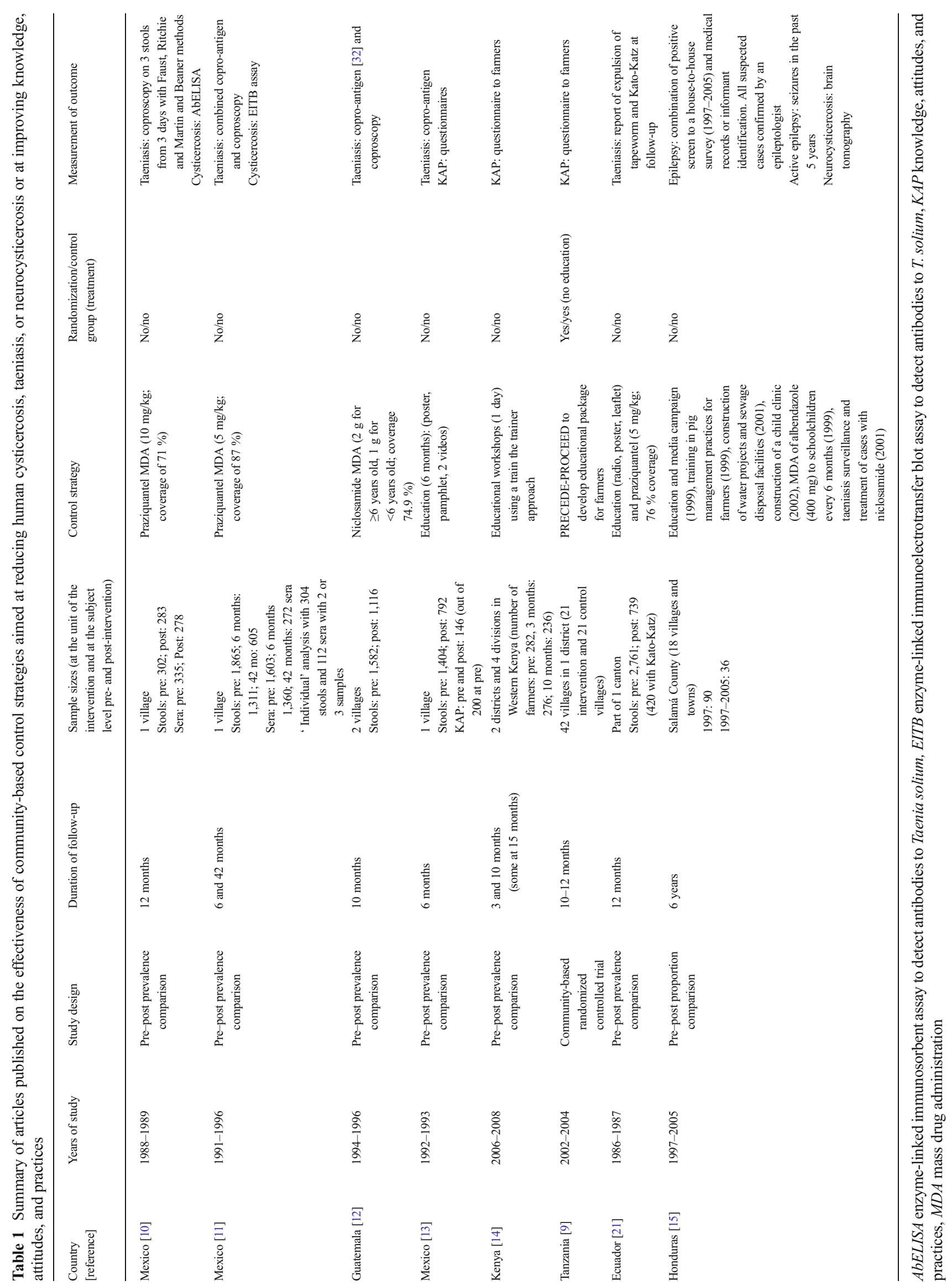




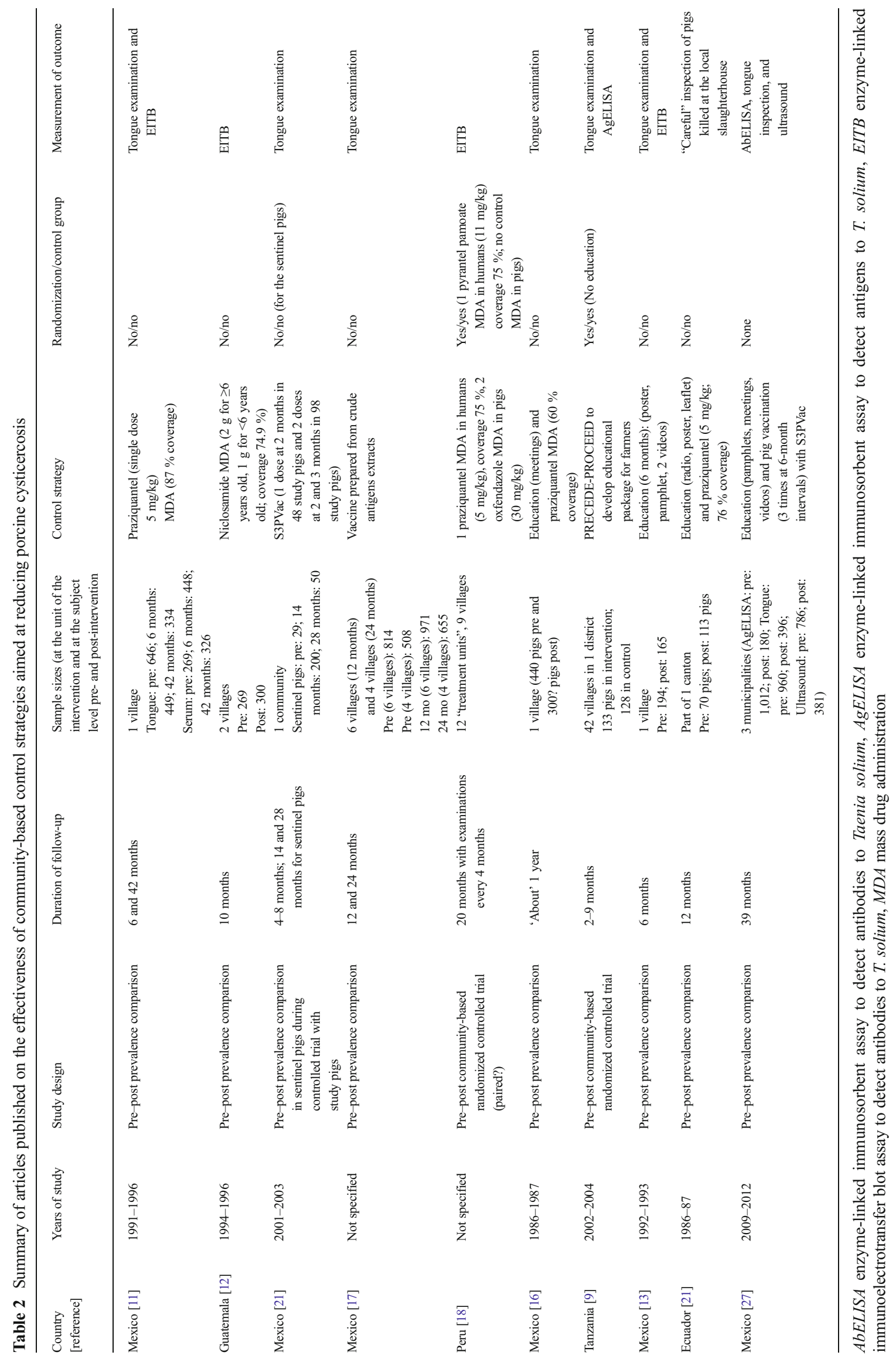




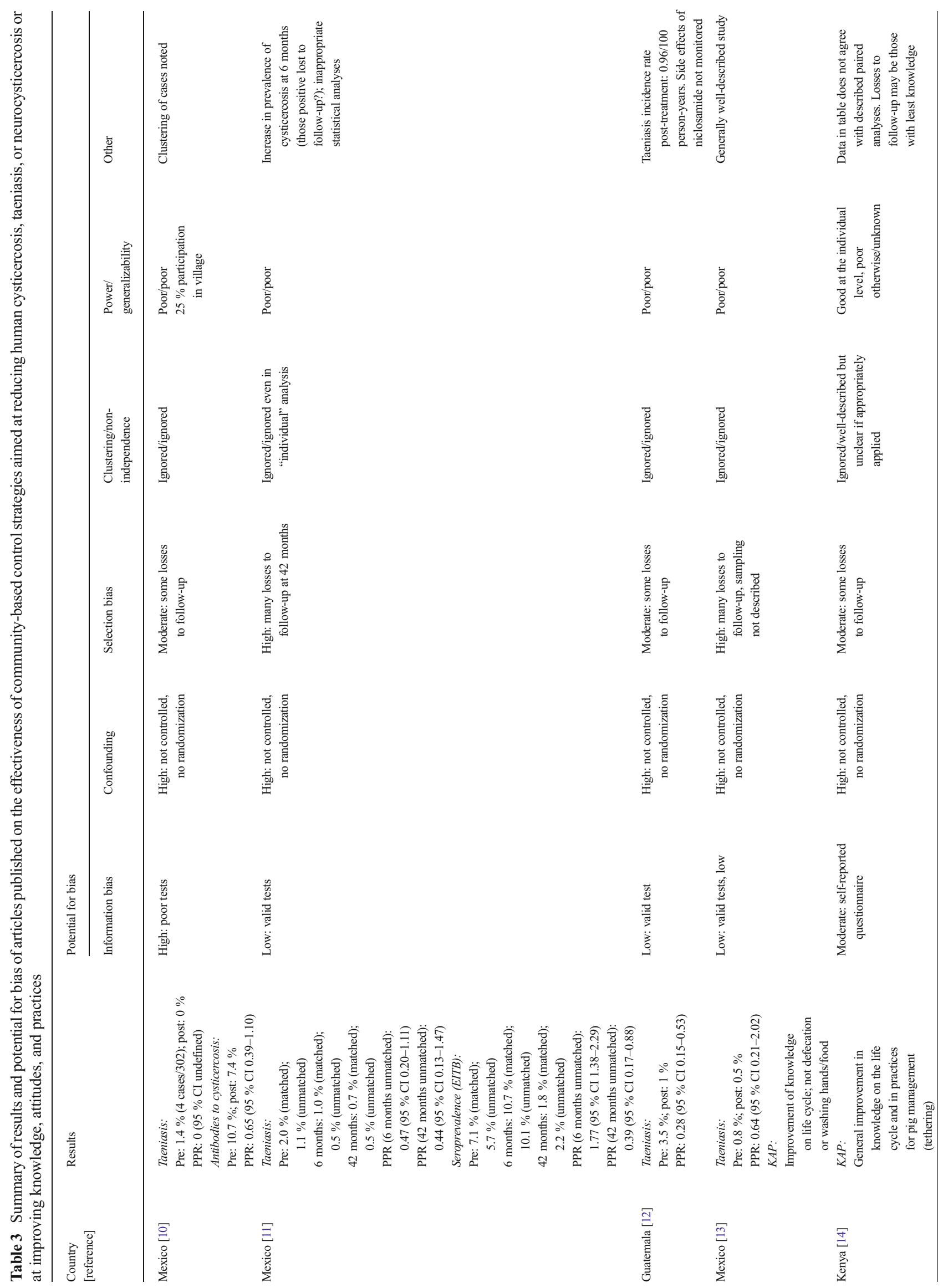


community level. The efficacy and safety of anti-helminthic drugs established in experimental studies were recently reviewed [37], but with little criticism and assessment of biases. The most recent experiment compared the efficacy of nine different regimens [38]. Although they have limitations, these studies suggest oxfendazole $(30 \mathrm{mg} / \mathrm{kg})$ to be the most effective drug to treat cysts in pig muscles, but less so in pig brains $[37,38]$.

We could only identify one randomized controlled trial estimating the effectiveness of oxfendazole in field conditions [39]. In this trial, 216 piglets aged 4 months were blockrandomized (blocked by litter) to receive one dose of oxfendazole $(30 \mathrm{mg} / \mathrm{kg})$ at 4 months (one piglet per litter), two doses at 4 and 9 months (one piglet per litter), or serve as controls (two piglets per litter). Oxfendazole reduced the incidence rate of infection, especially at older ages. Unfortunately, inappropriate statistical analyses were applied by ignoring the block randomization and clustering and using a logistic regression to analyze a common outcome [40]. This resulted in overestimating the magnitude and precision of the treatment effect. In addition, several piglets were lost to follow-up. If the reason for the loss was linked to the outcome in only one group, this could introduce a selection bias, the direction of which is difficult to predict. Acknowledging these limitations, one can conclude that oxfendazole is likely to reduce porcine cysticercosis, but to a lesser magnitude than was reported [39].

To summarize, despite abundant literature on the efficacy of anti-parasitic treatments for porcine cysticercosis, we were unable to find studies assessing the effectiveness of community-based delivery of porcine MDA alone on cysticercosis.

\section{Effectiveness of Pig Vaccination Alone}

Three major vaccines have been assessed in field studies, namely: (1) a vaccine using crude antigen extracts collected from metacestodes of infected pigs developed by the Molinari group [41]; (2) a recombinant oncosphere antigen-based TSOL16 or TSOL18 vaccine developed by the Lightowlers group [42]; and (3) a peptide-based S3PVac developed by the Sciutto group [43]. While TSOL18 has been tested for its effectiveness in field-based randomized controlled trials in Peru (with TSOL16) [44] and Cameroon [45], no implementation or measure at the community level has been assessed. The effect of both S3PVac [26] and the crude extract vaccine [22] have been assessed using community-level outcomes in Mexico, but, unfortunately, both used the poorly accurate tongue examination method for diagnosing porcine cysticercosis, making the interpretation of the results extremely difficult. Indeed, considering that tongue inspection has sensitivity and specificity values of around 50 and $80 \%$ [46], 


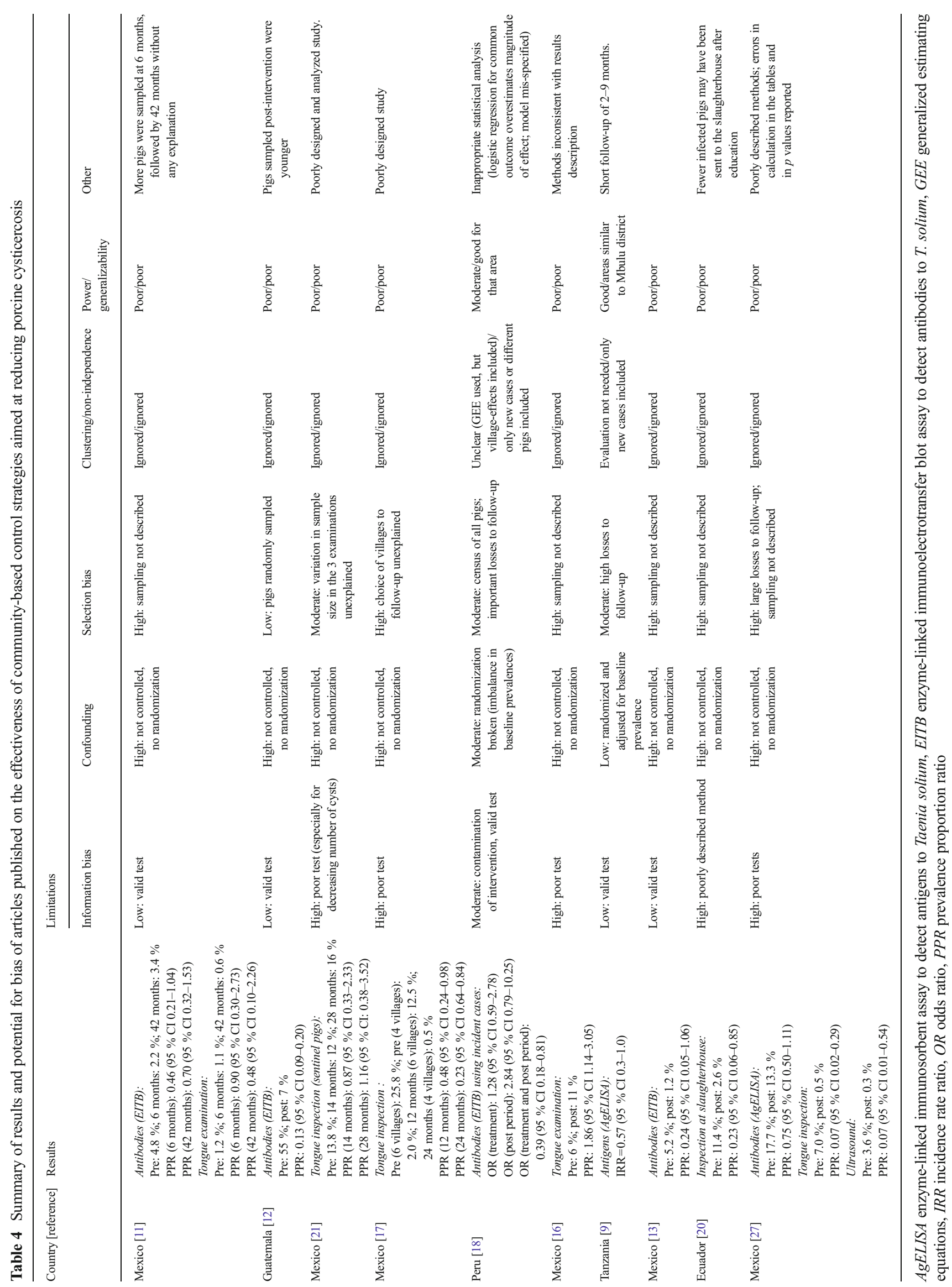


respectively, the direction of the bias is difficult to predict. This limitation is in addition to the lack randomization, control group, control for confounding, and possible selection bias. We can henceforth conclude that there is insufficient evidence to determine if porcine vaccination impacts the transmission of $T$. solium at the community level.

Several controlled trials have been conducted in the field with pigs being randomized (usually within a same litter) to receive a vaccine or placebo, and the outcome measured by the number of cysts or viable cysts found at necropsy [43-45, 47]. Three doses of TSOL18 (at baseline, 4 weeks, and 4 months) in combination with oxfendazole at 4 weeks, compared with a treatment with oxfendazole at 4 weeks, was very effective at eliminating any cysts in the vaccinated group, while $19.6 \%$ of the control group had muscle cysts (20 pigs with 337,080 cysts) [45]. In a similar trial, 137 pairs of piglets aged 2-4 months were randomized to receive either TSOL16-18 at baseline with a booster at 4 weeks combined with a swine classical fever vaccine or a swine classical vaccine only, and then necropsied 7 months later [44]. Of the 100 pairs necropsied, four had both piglets infected, 13 had control piglets infected and vaccinated piglets uninfected, and three had the vaccinated piglets infected and the control piglets uninfected. Most cysts found in the vaccination group were degenerated, in contrast to the viable cysts found in the control group. Both studies showed TSOL18 and TSOL16-18 to be effective vaccines at the pig level, as long as at least two doses with a 4-week interval were given. The S3PV vaccine also showed promising results [43, 47]. Muscle cysts were found in $7.5 \%$ of piglets vaccinated with two doses of S3PV (at 60 and 90 days of age) and in $15.8 \%$ of control piglets [43]. Despite these interesting results, the use of inappropriate statistical analyses makes the interpretation of the reduction in the number of cysts difficult. In another trial where 331 of 1,047 pigs randomized were autopsied, the odds of presenting cysts at necropsy was $2.3(95 \%$ CI 1.2-4.3) times higher in the control group than in the vaccinated group (two doses of S3PV at 3-4 and at 4-5 months of age), in a model including castration status and weight to adjust for factors associated with losses to follow-up. These randomized trials suggest that pig vaccination is effective at preventing infection, with TSOL16-18 appearing to show a higher efficacy than S3PV when two doses are used. However, the magnitude of the efficacy is difficult to assess with the data provided by the authors and the improper statistical analyses used in most cases. In addition, no evidence is available on the community-level effect of vaccinating pigs on human taeniasis and porcine and human cysticercosis. Pig vaccination remains problematic in terms of its sustainability in very poor areas where farmers cannot even afford to feed their pigs.

\section{Effectiveness of a Combination of Mass Drug Delivery of Anti-Parasitic Treatments to Pigs and Humans}

One pre-post community-based randomized controlled trial assessed the effectiveness of a program using MDA of oxfendazole ( $30 \mathrm{mg} / \mathrm{kg}$, two rounds at an unspecified interval) to pigs and of praziquantel $(5 \mathrm{mg} / \mathrm{kg}$, one round, $75 \%$ coverage) to humans [24]. The study divided nine endemic villages into 12 "treatment units" to increase the sample size, but, due to mistakes in implementation, seven units received the intervention, while five served as controls, with three villages being "split" into two units. In addition, one split village was missing baseline incidence rate estimates. The break in randomization resulted in an imbalance in the baseline prevalence of porcine cysticercosis, which may lead to an over-estimation of the intervention effectiveness. Similarly, the "split" strategy will likely result in contamination, leading to under-estimation of the effectiveness. The authors used a logistic regression to analyze incidence rate data, included each village as an independent factor, ignored clustering, and did not interpret the interaction term correctly. These improper analyses along with important issues with the study design make it impossible to accurately interpret the results of this study.

\section{Effectiveness Of Educational Programs Alone}

Education is recognized as an important tool in the control of cysticercosis [2-6, 24]. Three studies have assessed the effectiveness of education programs for pig farmers $[14,19]$ or the general population [18] on reducing human taeniasis [18] or porcine cysticercosis $[14,18]$, or improving knowledge, attitudes, and practices $[14,18,19]$. In a pre-post prevalence comparison study conducted in one Mexican village [18], an education program including the use of pamphlets, posters, and two videos designed following in-depth interviews led to a PPR of 0.64 (95\% CI 0.21-2.02) for taeniasis as measured by a copro-antigen assay 6 months post-intervention. This study suffers from the lack of a control group and randomization as well as a very high proportion of losses to follow-up.

The Mexican group also measured the change in porcine cysticercosis prevalence (194 at baseline and 165 at 6 months post-intervention) using EITB assay and obtained a PPR of 0.24 (95\% CI 0.05-1.06), but pig sampling was not described [18]. A much better designed community-based randomized controlled trial evaluating the effectiveness of a pig management educational program developed following a PRECEDEPROCEED approach was conducted in 42 villages of northern Tanzania [9]. An incidence rate ratio of 0.57 (95\% CI 0.3-1.0) 
for porcine cysticercosis (measured with antigen-ELISA [48] adjusted for baseline prevalence of porcine cysticercosis) was obtained. Among all the papers reviewed, this is the only paper that followed the CONSORT guidelines to report results of community-based randomized controlled trials [49]. These authors are also the only ones to conduct a cost analysis, demonstrating that the education program was cost beneficial to pig farmers, with a net present value of US\$3,507 (95\% CI 3,421-3,591) [50]. Nonetheless, this study suffers from two important limitations, namely a short median follow-up time (4 months) and a large proportion of losses to follow-up (52\%) resulting from a severe drought which forced farmers to sell or eat the pigs that they were given. This makes it impossible to assess the long-term impact of the intervention.

All three studies assessed the impact of their education program on knowledge, attitudes, and practices regarding cysticercosis $[14,18,19]$. The study in Kenya interviewed 296 pig farmers before and twice after receiving a workshop and one-to-one training using a train-the-trainer approach, but the number of farmers interviewed at each visit varied [19]. Only the Tanzanian study analyzed the data at the community level and was designed in such manner that the effect of monitoring alone could be determined [14]. Interestingly, monitoring alone had a strong effect on knowledge, meaning that farmers not living in intervention villages may have heard about it or that being given sentinel pigs made them improve their knowledge about cysticercosis. Since the Kenyan [19] and Mexican [18] studies did not have a control group, it is impossible to determine if the observed improvements in knowledge were due to monitoring alone. In Tanzania and Mexico, the only reported practices improved by the intervention were a reduction in eating contaminated pork and an increased practice of boiling drinking water $[14,18]$. In Kenya, there was a tendency for improvement in the proportion of farmers keeping their pigs tethered $100 \%$ of the time, but the results among those not lost to follow-up was inconclusive [19]. All of these studies suffer from a possible reporting bias for practices as research participants may report preferred behaviors instead of the truth, as noticed by Sarti et al. [18].

To summarize, education alone seems to reduce the level of porcine cysticercosis at least in the short-term, but the impact on knowledge and practices is less certain. Endemic areas for cysticercosis are generally very poor, which limits the ability of farmers to restrict pigs from roaming, and also may limit the ability to maintain latrines. Access to water could also limit improvement in hand washing. This probably explains the general lack of improvement in practices. Nonetheless, the presence of a research team appears to improve knowledge, which could improve practices in the longer term, but this will require longer follow-ups to take place.

\section{Effectiveness of Programs Combining Education Activities and Mass Drug Administration of Anti-Parasitic Treatments to Humans or Vaccination of Pigs}

Two studies combined education with human MDA to control cysticercosis $[20,25]$, while one combined education with porcine vaccination [27]. The first study was a pre-post proportion comparison study conducted in 18 villages in Salamá county (Honduras) that involved a very intense, multi-facetted intervention, spanning several years (see Table 1) [20]. The intervention was associated with a PPR of $0.38(95 \% \mathrm{CI}$ $0.16-0.89)$ in the proportion of epilepsy cases showing lesions of neurocysticercosis [20]. In addition to being a non-randomized, uncontrolled pre-post comparison study, only new-onset seizure cases were included post-intervention (from 1997 to 2005), while all cases of active epilepsy were included at baseline. This would lead to a higher prevalence of neurocysticercosis at baseline due to old calcified lesions still causing seizures, over-estimating the effect of the intervention. The second study, undertaken in Ecuador, implemented a shorter but still intense intervention where, during door-todoor visits, leaflets on cysticercosis/taeniasis control, verbal health education, and an offer for treatment with praziquantel $(5 \mathrm{mg} / \mathrm{kg})$ were provided to all individuals living in one canton of Ecuador [25]. Taeniasis was measured by self-report of parasite expulsion, an extremely unreliable measurement tool, especially without the use of a purgation agent, in addition to the possible under-reporting at follow-up. This design flaw invalidates the observed reduction in the prevalence of human taeniasis. The effect on porcine cysticercosis was assessed through a vaguely described "careful inspection" of pigs after slaughter at the Canton's slaughterhouse. Since education may teach farmers to assess infection by looking under pigs' tongues, owners of infected pigs may have been less inclined to send them to the official slaughterhouse after the intervention, which also invalidates the observed reduction in the prevalence of porcine cysticercosis. The third study, conducted in three municipalities of Mexico, was a pre-post prevalence comparison evaluating the effect of vaccinating all pigs aged more than 2 months with S3Pvac at least three times, with an educational program including meetings and distribution of educational pamphlets and videos about T. solium (27). The authors report a reduction in the prevalence using tongue inspection, ultrasound, and, to a lower extent, antibodyELISA, but the study suffers from many biases making it difficult to interpret the results. The poor designs of these three studies make it impossible to draw firm conclusions on 
the effect of combined interventions on the control of cysticercosis.

\section{Results from Theoretical Transmission Dynamics Models}

In the absence of convincing evidence from field trials, transmission dynamics models can be helpful in ranking the relative effectiveness of control strategies while taking the dynamics of the infection as well as herd immunity into account. A study modeling the transmission dynamics of $T$. solium between pigs and humans suggests that the most sustainable intervention remains the improvement of sanitation and pig management practices, but the effect of such improvement would need to be considerable [51]. This model is limited by its lack of age structure, seasonality, and possible clustering of infection.

\section{Epidemiological Methods Limitations of the Reviewed Studies and Conclusion}

All of the articles reviewed aimed at reducing human cysticercosis or taeniasis $[15-18,20,26]$ and all $[16-18,21,22$, 26-27], except two [14, 23], aimed at reducing porcine cysticercosis, lacked the use of randomization and of a control group, preventing control for confounding factors through study design or statistical methods. Randomization ensures that all confounding factors, measured or unmeasured, are well-balanced, preventing confounding bias. They are therefore susceptible to confounding bias, the direction and magnitude of which is difficult to predict. The use of a control group ensures that participation itself is not the cause of the change in the outcome over time. For instance, without a control group, any changes in environmental or behavioral factors over time may distort the true effectiveness of the intervention, leading to an overestimation of the effectiveness of the interventions. Indeed, improvement in education levels and pig management practices over time was acknowledged in one study [16] and the impact of monitoring (i.e., time) on knowledge was demonstrated in another [14]. The lack of a control group also prevents statistical adjustment for confounding. None of these studies, except for two [14, 23], had a sample size large enough to generate statistical power and generalize the results, with one village or community included where very intense interventions were offered. Such approaches are unlikely to be sustainable when used in larger settings with limited resources. The study of a single community also limits the assessment of potential variability in the effectiveness of the intervention. In addition, except for three $[14,16,23]$, no study acknowledged the non-independence of individuals studied pre- and post-intervention, leading to the use of improper statistical analyses and biased results. The studies conducted in Tanzania [14] and Peru [23] remain the best available, but unfortunately did not report on the effect of the intervention on human taeniasis or cysticercosis, and also had some limitations.

We can conclude that there is insufficient evidence to suggest any community-based intervention to control human and porcine cysticercosis. Given this lack of evidence, it is very unlikely for the infection to be eradicated from poor endemic areas in the foreseeable future. Future studies should carefully follow recommendations provided in by Thompson and co-workers [52] for the conduct of community-based controlled randomized trials, as well as adhere to the CONSORT guidelines when reporting the results [49]. This will considerably improve the evidence on the effectiveness of alternative control strategies for T. solium at the community level and hopefully lead to the implementation of costeffective control strategies.

Acknowledgments This work was conducted with the support of the National Institute of Neurological Disorders and Stroke (NINDS) and of the Forgarty International Center (FIC) of the National Institutes of Health (NIH) under the Brain Disorders in the Developing World: Research Across the Lifespan program, grant R01NS064901.

\section{Compliance with Ethics Guidelines}

Conflict of Interest Hélène Carabin and Aminata A. Traoré declare that they have no conflicts of interest.

Human and Animal Rights and Informed Consent This article does not contain any studies with human or animal subjects performed by any of the authors.

\section{References}

Papers of particular interest, published recently, have been highlighted as:

-. Of major importance

1. Centers for Disease Control and Prevention. Recommendations of the International Task Force for Disease Eradication. MMWR Recomm Rep. 1993;42:1-38.

2.• The Carter Center. Summary of the twenty-first meeting of the international task force for disease eradication (II). July 10, 2013. http://www.cartercenter.org/resources/pdfs/news/health publications/itfde/ITFDE-summary-071013.pdf. Accessed 6 Au 2014. This report is of importance because it emphasizes for the first time the key role of human hygiene and sanitation as a promising strategy to control cysticercosis.

3. Willingham 3rd AL, Wu HW, Conlan J, Satrija F. Combatting Taenia solium cysticercosis in Southeast Asian and opportunity for improving human health and livestock production. Adv Parasitol. 2010;72:235-66.

4. Murrell KD (Ed.). WHO/FAO/OIE Guidelines for the Surveillance, Prevention and Control of Taeniosis/Cysticercosis. 2005. Office International des Epizooties, Paris. 
5. O’Neil SE, Winthrop KL, Gonzalez AE. Cysticercosis control: bringing advances to the field. J Glob Infect Dis. 2011;3: 156-60.

6. Fleury A, Sciutto E, de Aluja AS et al. Control of Taenia solium transmission of taeniosis and cysticercosis in endemic countries: the roles of continental networks of specialists and of local health authorities. From: Foyaca-Sibat H (Ed). Novel Aspects on Cysticercosis and Neurocysticercosis. InTech 2013. doi: 10.5772/ 51286. http://www.intechopen.com/books/novel-aspects-oncysticercosis-and-neurocysticercosis/control-of-taenia-soliumtransmission-of-taeniosis-and-cysticercosis-in-endemic-countriesthe-roles. Accessed 6 Aug 2014.

7. Flisser A. Taeniasis cysticercosis due to Taenia solium. In: Sun T, editor. Progress in Clinical Parasitology. New York: CRC Press; 1994. p. 77-116.

8. Pawlowski Z, Murrell KD. Taeniasis and cysticercosis. In: Hui YH, Sattar SA, Murrell KD, Nip WK, Stanfield PS, editors. Volume 2: Viruses, Parasites, Pathogens and HAACP. 2nd ed. New York: Marcel Dekker, Inc; 2000. p. 217-27.

9. Maya C, Torner-Morales FJ, Lucario ES, Hernandez E, Jumenez B. Viability of six species of larval and non-larval helminth eggs for different conditions of temperature, $\mathrm{pH}$ and dryness. Water Res. 2012;46:4770-82.

10. Flisser A. Neurocysticercosis in Mexico. Parasitol Today. 1988;4: 131-7.

11. Pouedet MSR, Zoli AP, Nguekam L, Vondou L, Assana E, Speybroeck N, et al. Epidemiological survey of swine cisticercosis in two rural communities of West-Cameroon. Vet Parasitol. 2002;106:45-54.

12. Shey-Njila O, Zoli PA, Awah-Ndukum J, Nguekam JP, Assana E, Byambas $\mathrm{P}$, et al. Porcine cysticercosis in village pigs of northwest Cameroon. J Helminthol. 2003;77:351-4.

13. Carabin H, Ndimubanzi PC, Budke CM, et al. Clinical manifestations associated with neurocysticercosis : a systematic review. PLoS Negl Trop Dis. 2011;5:e1152.

14. Ngowi HA, Carabin H, Kassuku AA, et al. A health-education intervention trial to reduce porcine cysticercosis in Mbulu District, Tanzania. Prev Vet Med. 2008;85:52-67.

15. Diaz Camacho SP, Candil Ruiz A, Suate Peraza V, et al. Epidemiologic study and control of Taenia solium infections with praziquantel in a rural village of Mexico. Am J Trop Med Hyg. 1991;45:522-31.

16. Sarti E, Schantz PM, Avilla G, Ambrosio J, Medina-Santillan R, Flisser A. Mass treatment against human taeniasis for the control of cysticercosis: a population-based intervention study. Trans R Soc Trop Med Hyg. 2000;94:85-9.

17. Allan JC, Velasquez-Tohom M, Fletes C, et al. Mass chemotherapy for intestinal Taenia solium infection: effect on prevalence in humans and pigs. Trans R Soc Trop Med Hyg. 1997;91:595-8.

18. Sarti E, Flisser A, Schantz PM, et al. Development and evaluation of a health education intervention against Taenia solium in a rural community in Mexico. Am J Trop Med Hyg. 1997;56:127-32.

19. Wohlgemut J, Dewey C, Levy M, Mutua F. Evaluating the efficacy of teaching methods regarding prevention of human epilepsy caused by Taenia solium neurocysticercosis in Western Kenya. Am J Trop Med Hyg. 2010;82:634 42.

20. Medina MT, Aguilar-Estrada RL, Alvarez A, et al. Reduction in rate of epilepsy from neurocysticercosis by community interventions: the Salamá, Honduras study. Epilepsia. 2011;52:1177-85.

21. Keilbach NM, de Aluja AS, Sarti-Gutierrez E. A programme to control taeniasis-cysticercosis (T. solium): experiences in a Mexican village. Acta Leiden. 1989;57:181-9.

22. Molinari JL, Rodríguez D, Tato $\mathrm{P}$, et al. Field trial for reducing porcine Taenia solium cysticercosis in Mexico by systematic vaccination of pigs. Vet Parasitol. 1997;69:55-63.
23. Garcia HH, Gonzalez AE, Gilman RH, et al. Combined human and porcine mass chemotherapy for the control of T. solium. Am J Trop Med Hyg. 2006;74:850-5.

24. Garcia HH, Gonzalez AE, Rodriguez S, et al. Epidemiology and control of cysticercosis in Peru [in Spanish]. Rev Peru Med Exp Salud Publica. 2010;27:592-7.

25. Cruz M, Davis A, Dixon H, Pawlowski ZS, Proano J. Operational studies on the control of Taenia solium taeniasis/cysticercosis in Ecuador. Bull World Health Organ. 1989;67:401-7.

26. Sciutto E, Morales J, Martinez JJ, et al. Further evaluation of the synthetic peptide vaccine S3Pvac against Taenia solium cysticercosis in pigs in an endemic town of Mexico. Parasitology. 2007;164: 124-33.

27. De Aluja AD, Suarez-Martin R, Sciutto-Conde E, et al. Evaluacion del impacto de un programa de control de la teniasis-cisticercosis. Salud Pública Méx. 2014;56:259-65.

28. Wu W, Qian X, Huang Y, Hong Q. A review of the control of Clonorchiasis sinensis and Taenia solium taeniasis/cysticercosis in Chin. Parasitol Res. 2012;111:1879-84.

29. World Health Organization. WHO Model Prescribing Information. Drugs used in parasitic diseases. WHO (Geneva) 1995. Second Edition.

30. Bustos JA, Rodrigues S, Jimenez JA. Detection of Taenia solium taeniasis coproantigen is an early indicator of treatment failure for taeniasis. Clin Vaccine Immunol. 2012;19:570-3.

31. Pawlowski ZS. Efficacy of low doses of praziquantel in taeniasis. Acta Trop. 1991;48:83-8.

32. Allan JC, Avila G, Garcia-Noval J, Flisser A, Craig P. Immunodiagnosis of taeniasis by coproantigen detection. Parasitol. 1990;101:473-7.

33. Praet N, Verweii JJ, Meape KE. Bayesian modelling to estimate the test characteristics of coprology, coproantigen ELISA and a novel real-time PCR for the diagnosis of taeniasis. Trop Med Int Health. 2013;18:608-14.

34. Tsang VC, Brand HA, Boyer AE. An enzyme-linked immunoelectrotransfer blot assay and glycoprotein antigens for diagnosing human cysticercosis (Taenia solium). J Infect Dis. 1989;159:50-9.

35. Larralde C, Lacelette JP, Madrazo I, et al. Reliable serology of Taenia solium cysticercosis with antigens from cyst vesicular fluid: ELISA and hemagglutination tests. Am J Trop Med Hyg. 1986;35: 965-73.

36. Praet N, Rodriguez-Hidalgo R, Speybroeck N, et al. Infection with versus exposure to Taenia solium: what do serological test results tell us? Am J Trop Med Hyg. 2010;83:413-5.

37. Mkupasi EM, Sikasunge CS, Ngowi HA, Vang Johansen M. Efficacy and safety of anthelmintics tested against Taenia solium cysticercosis in pigs. PLoS Negl Trop Dis. 2013;7: e2200.

38. Gonzalez AE, Bustos JA, Jimenez JA, et al. Efficacy of diverse antiparasitic treatments for cysticercosis in the pig model. Am J Trop Med Hyg. 2012;87:292-6.

39. Pondja A, Neves L, Mlangwa J, et al. Use of oxfendazole to control porcine cysticercosis in a high-endemic area of Mozambique. PLoS Negl Trop Dis. 2012;6:e1651.

40. McNutt LA, Wu C, Xue X, Hafner JP. Estimating the relative risk in cohort studies and clinical trials of common outcomes. Am J Epidemiol. 2003;157:940-3.

41. Molinari JL, Soto R, Tato P, et al. Immunization against porcine cysticercosis in an endemic area in Mexico: a field and laboratory study. Am J Trop Med Hyg. 1993;49:502-12.

42. Lightowlers MW. Eradication of Taenia solium cysticercosis: a role for vaccination of pigs. Int J Parasitol. 2010;40:1183-92.

43. Huerta M, de Aluja AS, Fragoso G, et al. Synthetic peptide vaccine against Taenia solium pig cysticercosis: successful vaccination in a controlled field trial in rural Mexico. Vaccine. 2001;20:262-6. 
44. Jayashi CM, Kyngdon CT, Gauci CG. Successful immunization of naturally reared pigs against porcine cysticercosis with a recombinant oncosphere antigen vaccine. Vet Parasitol. 2012;188:261-7.

45. Assana E, Kyngdon CT, Gauci CG. Elimination of Taenia solium transmission to pigs in a field trial of the TSOL18 vaccine in Cameroon. Int J Parasitol. 2010;40:515-9.

46. Krecek RC, Michael LM, Schantz PM, et al. Prevalence of Taenia solium cysticercosis in swine from a community-based study in 21 villages of the Eastern Cape Province, South Africa. Vet Parasitol. 2008;154:38-47.

47. Morales J, Martínez JJ, Manoutcharian K, et al. Inexpensive anticysticercosis vaccine: S3Pvac expressed in heat inactivated M13 filamentous phage proves effective against naturally acquired Taenia solium porcine cysticercosis. Vaccine. 2008;26:2899-905.

48. Dorny P, Phiri IK, Vercruysse J, et al. A Bayesian approach for estimating values for prevalence and diagnostic test characteristics of porcine cysticercosis. Int $\mathrm{J}$ Parasitol. 2004;34:569-76.

49. Campbell MK, Piaggio G, Elbourne, Altman DG, for the CONSORT Group. Consort 2010 statement: extension to cluster randomised trials. BMJ. 2012;345:e5661.

50. Ngowi HA, Mlangwa JED, Carabin $\mathrm{H}$ et al. Financial efficiency of health and pig management education intervention in controlling porcine cysticercosis in Mbulu District, northern Tanzania. Livestock Res Rural Dev 2007; 19. http://www.lrrd.org/lrrd19/5/ ngow19062.htm. Accessed 20 Aug 2014.

51. Kyvsgaard NC, Johansen MV, Carabin H. Simulating transmission and control of Taenia solium infections using a Reed-Frost stochastic model. Int J Parasitol. 2007;37:547-58.

52. Thompson B, Coronado G, Snipes SA, Puschel K. Methodological advances and ongoing challenges in designing community-based health promotion programs. Ann Rev Publ Health. 2003;24:315-40. 